\title{
Chest radiographs and BNP levels provided complementary information beyond clinical findings for diagnosing heart failure
}

Knudsen CW, Omland T, Clopton P, et al. Diagnostic value of B-type natriuretic peptide and chest radiographic findings in patients with acute dyspnea. Am J Med 2004;116:363-8.

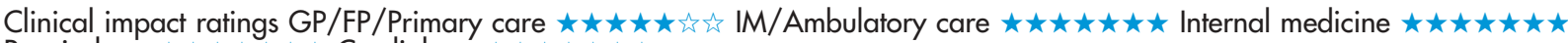

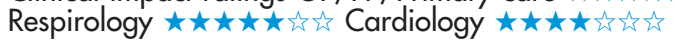

In patients with acute dyspnoea, how do chest radiographic findings and circulating B type natriuretic peptide (BNP) levels compare for diagnosing heart failure (HF)?

\section{METHODS}

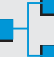

Design: blinded comparison of chest radiographs and BNP levels with confirmatory clinical diagnosis.

Setting: 5 teaching hospitals in the United States and 2 in Europe.

Patients: 880 patients (mean age $64 \mathrm{y}, 55 \%$ men) presenting to the emergency department (ED) with a principal complaint of shortness of breath (either the sudden onset of dyspnoea with no history of chronic dyspnoea or an increase in the severity of chronic dyspnoea); and had complete information on BNP, historical, clinical, and electrocardiographic data, and chest radiographic findings. Patients with dyspnoea not caused by HF (eg., stabbing injuries, trauma, and pneumothorax) were excluded.

Description of tests: chest radiographs were obtained in the ED 101| and the presence of cardiomegaly, cephalisation, interstitial oedema, alveolar oedema, pleural effusion, hyperinflated lungs, and pneumonic infiltrates, as interpreted by a radiologist, was recorded. During initial evaluation, BNP levels were measured using the Triage BNP test (Biosite Diagnostics, San Diego, CA, USA), a fluorescence immunoassay for the quantitative determination of BNP in whole blood and plasma specimens. BNP levels were analysed within 4 hours or were centrifuged, frozen, and analysed 1 to 2 days later.

Diagnostic standard: about 30 days after the ED visit, the results of electrocardiography, chest radiography, echocardiography, clinical test results, consultations, and medical record information were used by 2 independent cardiologists to categorise cases as caused by acute $\mathrm{HF}$ or having noncardiac causes.

Outcomes: sensitivity, specificity, and positive and negative likelihood ratios for the diagnosis of acute HF.

\section{MAIN RESULTS}

447 of 880 patients $(51 \%)$ had a final diagnosis of acute HF. Of these, $90 \%$ had BNP levels $\geqslant 100 \mathrm{pg} / \mathrm{ml}$. 576 of 880 patients (66\%) fulfilled

For correspondence: Dr T Omland, University of Oslo, Oslo, Norway. torbjorn.omland@klinmed.vio.no

Source of funding: Biosite Diagnostics. the Framingham criteria for HF. The table shows the results. In a multivariate analysis, additional information beyond the clinical predictors of acute HF was provided by BNP at a cutpoint of $\geqslant 100 \mathrm{pg} /$ $\mathrm{ml}$ (odds ratio [OR] 12.3, 95\% CI 7.4 to 20.4) and chest radiographic variables of cardiomegaly (OR 2.3, CI 1.4 to 3.7), cephalisation (OR 6.4, CI 3.3 to 12.5), and interstitial oedema (OR 7.0, CI 2.9 to 17).

\section{CONCLUSION}

In patients with acute dyspnoea, chest radiographic variables and circulating B type natriuretic peptide levels provided complementary diagnostic information beyond clinical predictors for diagnosing heart failure.

Abstract and commentary also appear in ACP Journal Club.

\section{Commentary}

$\Lambda$

rapid and accurate investigation of the symptom of acute shortness of breath is vital. Clinical and chest radiographic findings have long been used to diagnose HF. Echocardiograms are now also used frequently. Recently, BNP levels have been proposed to add diagnostic power in patients with acute dyspnoea. ${ }^{1} \mathrm{~A}$ series of studies has tested the utility of BNP in the emergency diagnosis of HF. ${ }^{2}{ }^{3}$ The study by Knudsen et al compared the diagnostic value of BNP levels with chest radiographs as adjuncts to clinical findings. Unfortunately, 706 patients were excluded from the original cohort $(n=1586)$ because they lacked complete information.

Knudsen et al found that both chest radiographic variables and BNP levels provide complementary diagnostic information beyond clinical predictors. The sensitivity of BNP at a low cutpoint $(100 \mathrm{pg} / \mathrm{ml})$ compared favourably with chest radiographic findings. This means that BNP levels could be used to rule out HF. However, the specificity of cephalisation and interstitial oedema was higher than even the highest BNP cutpoint $(400 \mathrm{pg} / \mathrm{ml})$. Therefore, some of the classic radiographic findings can better rule in HF.

Hence, BNP levels should not be used instead of chest radiography, because the diagnostic value of both tests is complementary. As pointed out by Knudsen et al, the diagnosis of HF should not be based on a single test. A final study with point of care radiographic interpretation and rapid BNP results would be valuable.

Peter Henriksson, MD, PhD

Karolinska Institute at Danderyd University Hospital, Stockholm, Sweden 1 Mukoyama M, Nakao K, Hosoda K, et al. J Clin Invest 1991 :87:1402-12.

2 Maisel AS, Krishnaswamy P, Nowak RM, et al. N Engl J Med 2002;347:161-7.

3 McCullough PA, Nowak RM, McCord J, et al. Circulation 2002;106:41622.

Diagnostic characteristics of chest radiographic findings and B type natriuretic peptide (BNP) levels for detecting heart failure*

\begin{tabular}{lllll}
\hline Tests & Sensitivity (95\% Cl) & Specificity (CI) & +LR & - LR \\
\hline Cardiomegaly & $79 \%(75$ to 83$)$ & $80 \%(76$ to 84$)$ & 3.98 & 0.26 \\
Cephalisation & $41 \%(37$ to 46$)$ & $96 \%(93$ to 97$)$ & 9.41 & 0.61 \\
Intersitial oedema & $27 \%(23$ to 31$)$ & $98 \%(96$ to 99$)$ & 12.67 & 0.72 \\
BNP (pg/ml) & $90 \%(86$ to 92$)$ & $75 \%(71$ to 79$)$ & 3.66 & 0.14 \\
$\quad \geqslant 100$ & $80 \%(76$ to 84$)$ & $87 \%(83$ to 90$)$ & 6.08 & 0.23 \\
$\geqslant 200$ & $71 \%(67$ to 75$)$ & $90 \%(87$ to 93$)$ & 7.18 & 0.32 \\
$\geqslant 300$ & & &
\end{tabular}

*Diagnostic terms defined in glossary; LRs calculated from data in article; Cls provided by author 